\title{
Equation of State Measurements at Extreme Pressures Using Laser-Driven Shocks
}

\author{
R. Cauble, L. B. Da Silva, P. M. Celliers, \\ G. W. Collins, D. M. Gold
}

This paper was prepared for submittal to the Proceedings to the Joint Institute for Fusion Theory

High Field Science Workshop

Livermore, CA

November 23-24, 1998

December 3, 1998

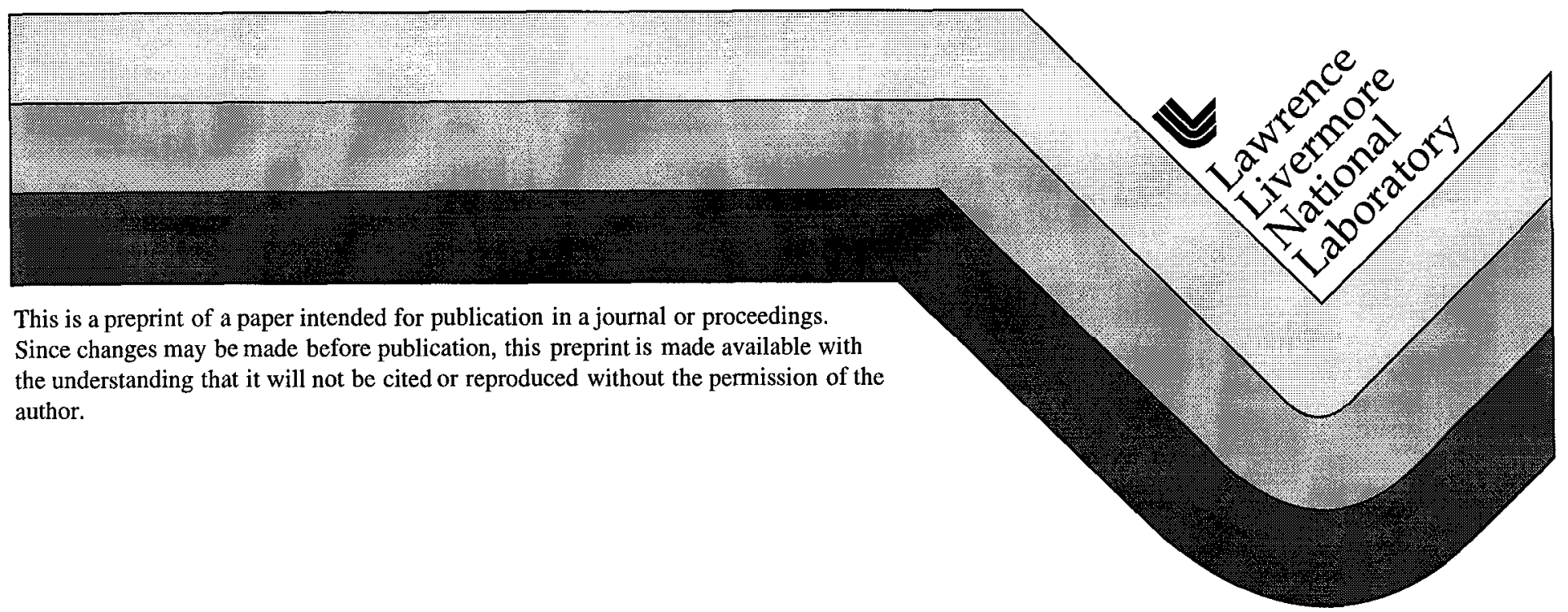




\section{DISCLAIMER}

This document was prepared as an account of work sponsored by an agency of the United States Government. Neither the United States Government nor the University of California nor any of their employees, makes any warranty, express or implied, or assumes any legal liability or responsibility for the accuracy, completeness, or usefulness of any information, apparatus, product, or process disclosed, or represents that its use would not infringe privately owned rights. Reference herein to any specific commercial product, process, or service by trade name, trademark, manufacturer, or otherwise, does not necessarily constitute or imply its endorsement, recommendation, or favoring by the United States Government or the University of California. The views and opinions of authors expressed herein do not necessarily state or reflect those of the United States Government or the University of California, and shall not be used for advertising or product endorsement purposes. 


\title{
Equation of State Measurements at Extreme Pressures using Laser-Driven Shocks
}

\author{
R. Cauble, L. B. Da Silva, P. M. Celliers, \\ G. W. Collins, and D. M. Gold
}

Lawrence Livermore National Laboratory, Livermore CA 94550 USA

\section{INTRODUCTION}

Laser-driven shock waves have long been a laboratory option for exploring material properties at extreme pressures, but the use of lasers for this purpose has been limited. Direct irradiation of a solid by even a tabletop laser focused to a small spot can produce a multi-Mbar shock. Although this has been known for several decades, during that time most laser-driven shock experiments have been demonstrations of high velocity shock waves, not the use of the shocks for measurements of, say, of the equation of state (EOS) of the shocked matter [1].

The main difficulties with laser-driven shock research have had to do with the technique itself. First, laser irradiation of a material instantly produces a hot plasma that can be the source of penetrating $x$ rays and high energy electrons. These can be absorbed by the sample prior to measurement, changing the initial conditions in the sample. Sample preheat is a problem that must be considered in all shock wave experiments, but it is especially troublesome for the high plasma temperatures and small dimensions typical of laser experiments. The small dimensions $(\sim 1 \mathrm{~mm})$ and short timescales (few ns) of laser experiments reveal the second difficulty, that of accurately diagnosing shocks moving at $15-50 \mathrm{~km} / \mathrm{s}$ across a span of only a few hundred microns. Diagnostics capable of few-micron spatial and few-ps temporal resolution are necessary. The third difficulty with laser experiments is driver energy. Moderate energy lasers can produce irradiation intensities of $10^{14} \mathrm{~W} / \mathrm{cm}^{2}$. This produces a hot plasma that ablates off the surface, driving a shock into the target. This intensity is sufficient to drive a $\sim 10 \mathrm{Mbar}$ shock. However, if the laser energy is low, the irradiation spot must be small and the beam sustained only for a short period. The resulting shock may possess strong curvature and a rapid decay time making it unusable for EOS measurements. However, international programs directed at obtaining electrical energy from the compression of deuterium-tritium pellets via inertially confined fusion (ICF) have pushed the construction of high-energy lasers and the development of fast, high-resolution diagnostics. Thus 
one result of ICF prograns is the capability to study materials at high density and temperature in the laboratory.

Two basic techniques are used in laser-driven shock wave research, direct and indirect drive. Irradiating a target with one or more laser beams is commonly referred to as direct drive. The relationship between intensity $I$ and pressure is given by $P(\mathrm{Mbar}) \approx$ few times $\left[I\left(10^{14}\right.\right.$ $\left.\left.\mathrm{W} / \mathrm{cm}^{2}\right) / \lambda(\mu \mathrm{m})\right]^{2 / 3}$. The indirect drive technique uses a high-atomic-number hohlraum into which usually several laser beams are focused. The laser light is absorbed and re-emitted from the interior hohlraum wall and then scatters many times within the cavity. The resulting $x$-rays, contained in the hohlraum and characterised by a radiation temperature $T_{\text {, }}$, are then used as the driving source. The pressure generated in the hohlraum is $\approx 10^{4} T_{r}(\mathrm{keV})^{3.5}$ Mbar. Typical gold hohlraum temperatures on the Nova laser at Lawrence Livermore National Laboratory [2] are 150 $-250 \mathrm{eV}$ producing radiation pressures of $\sim 100$ Mbar over periods of $1-2 \mathrm{~ns}$.

Very recently both methods have been used to obtain Hugoniot data using the impedance match technique (explained below). Direct drive impedance match data exist for iron [3] and gold [4]. Impedance match experiments with indirect drive were able to extend the Hugoniot of copper to $20 \mathrm{Mbar}$ [5]. The application of both methods to copper is discussed in Ref. 6 .

Both direct and indirect drive can be used to accelerate a small foil and collide the foil with a target, creating a shock in the target that is similar to the well-known high-explosive-driven and gas-gun-accelerated flyer plate experiments. There is a considerable advantage in utilising this technique: the drive energy is efficiently stored in the flyer and released suddenly as kinetic energy in collision. The highest planar shock pressure recorded in the laboratory - $750 \mathrm{Mbar}-$ was created this way [7]. Experience with thin directly-driven flyers has shown that small spatial nonuniformities in the laser beam can break up the foil [8]; this is a problem for EOS experiments.

\section{THE EOS AND THE PRINCIPAL HUGONIOT}

The principal Hugoniot is the locus of thermodynamic states in a material after the passage of a single shock. It is often portrayed as a plot of pressure $v s$. density but other representations are possible. Conservation relations require that two independent parameters be measured to obtain an absolute EOS data point. Impedance match experiments, that are generally easier to perform, produce relative EOS data. The conservation (or Hugoniot) equations relate the shock speed $U_{S}$, the particle speed $U_{p}$, the pressure $P$, and the final density $\rho$ are related by

and

$$
P-P_{o}=\rho_{O} U_{s} U_{p}
$$

$$
\rho / \rho_{O}=U_{s} /\left(U_{s}-U_{p}\right)
$$

where $\rho_{O}$ is the initial density, $P_{O}$ is the initial pressure and $\rho / \rho_{O}$ is the compression [9]. The particle speed is the speed that the shocked material, initially at zero velocity, has been accelerated to by the shock. Temperature is not a quantity in the Hugoniot relations; separate measurements are required for temperature determinations of shocked matter.

It is often relatively simple to measure shock speeds, even in laser experiments, although care must be taken to ensure sufficient accuracy. In impedance match experiments, two conjoined materials, the sample and a standard, are simultaneously shocked and shock speeds measured in each material. Since the EOS of the standard is assumed to be known (from calculations), the measured standard shock speed determines the state of the standard. From the measured speeds and the calculated standard EOS, a point on the Hugoniot of the sample can de determined relative to that of the standard. This is the method used in most measurements of equations of state shocked to megabar pressures. Most such experiments have been performed using a nuclear explosion to drive the strong shock [10]. 
Laser (indirecl) drive has been successfully used for impedance match measurements of copper up to $20 \mathrm{Mbar}$, conditions comparable to nuclear-driven shock experiments [5]. Indirect drive has also been used to obtain absolute Hugoniot data for beryllium up to 14 Mbar and polystyrene up to 40 Mbar. [11] The laser data confirmed nuclear-driven impedance match data for Be. However, the laser data found that an accepted polystyrene EOS was too compressible above 10 Mhar. In the experiments described in the following section, direct drive was utilized to examine the EOS of hydrogen isotopes. There too, surprises were found.

\section{EOS EXPERIMENTS ON HYDROGEN ISOTOPES AT MBAR PRESSURES}

The metallic transition in hydrogen and its effects on the equation of state at pressures near one Mbar are important for models of many astrophysical objects, including the Jovian planets [12] and low mass stars [13], as well as the design of deuterium-tritium-burning targets for inertial confinement fusion [14]. Fig. 1 shows the phase space of hydrogen in the vicinity of the finite-

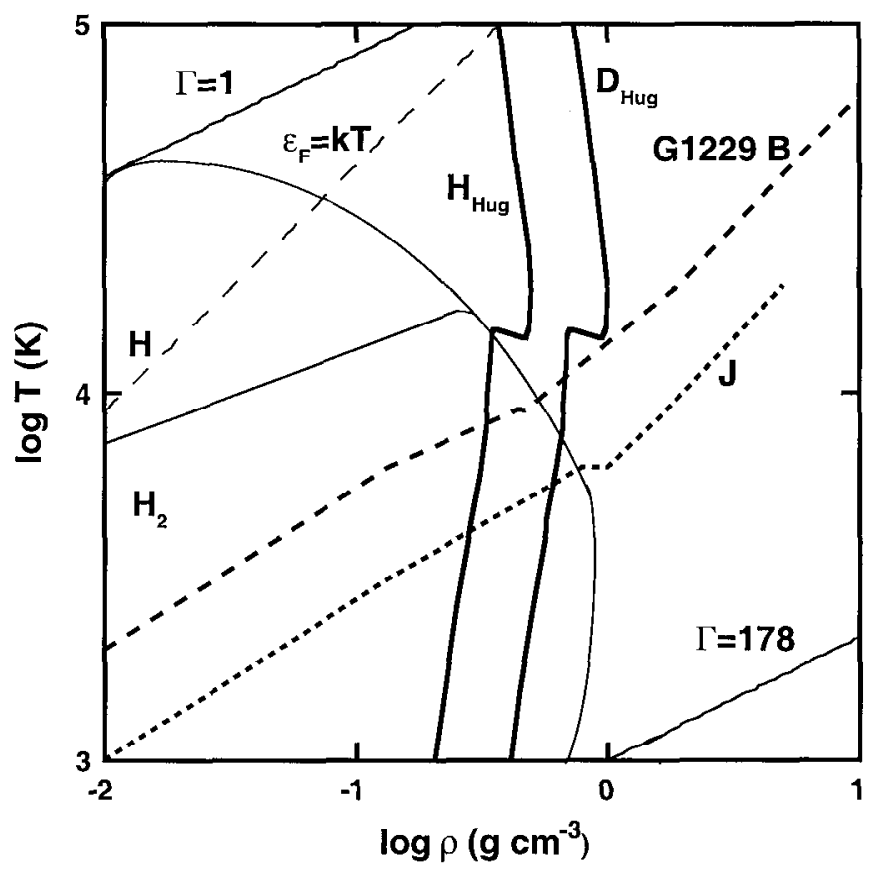

Figure 1. Model phase diagram of hydrogen [29] in the regime of the fluid metal-insulator phase transition. $\Gamma=e^{2}(4 \pi / 3 n)^{1 / 3} / k T$, where $n$ is the particle density, is a measurc of the interparticlc correlation strength. The Fermi energy is $\varepsilon_{F}$. "H ${ }_{2}$ " and " $\mathrm{H}$ " are regions that are mainly molecular and atomic hydrogen respectively; outside of these regions hydrogen is primarily an ionizcd fluid. "J" is a model isentrope for Jupiter [29]; "G1229 B" is an isentrope for brown dwarf G1229 B [30]. "H $\mathrm{H}_{\mathrm{Ilug}}$ " and " $\mathrm{D}_{\mathrm{Hug}}$ " are model hydrogen and deuterium Hugoniots [22]. 
temperature insulator-metal transition. This regime of high density and extreme pressure is very difficult to approach theoretically since it is a strongly correlated, partially degenerate composite of molecules, atoms, and electrons. For this reason, a number of theoretical models of the EOS of hydrogen have been proposed [15-22]. This makes reliable experimental data essential as a guide to thcory. We have accessed this regime by shocking liquid $\mathrm{D}_{2}$ to pressures at and above the metallic transition where we measured the thermodynamic properties of the shocked state.

Drive

Beam



Figure 2. Diagram of the cryogenic cell used in the EOS experiments.

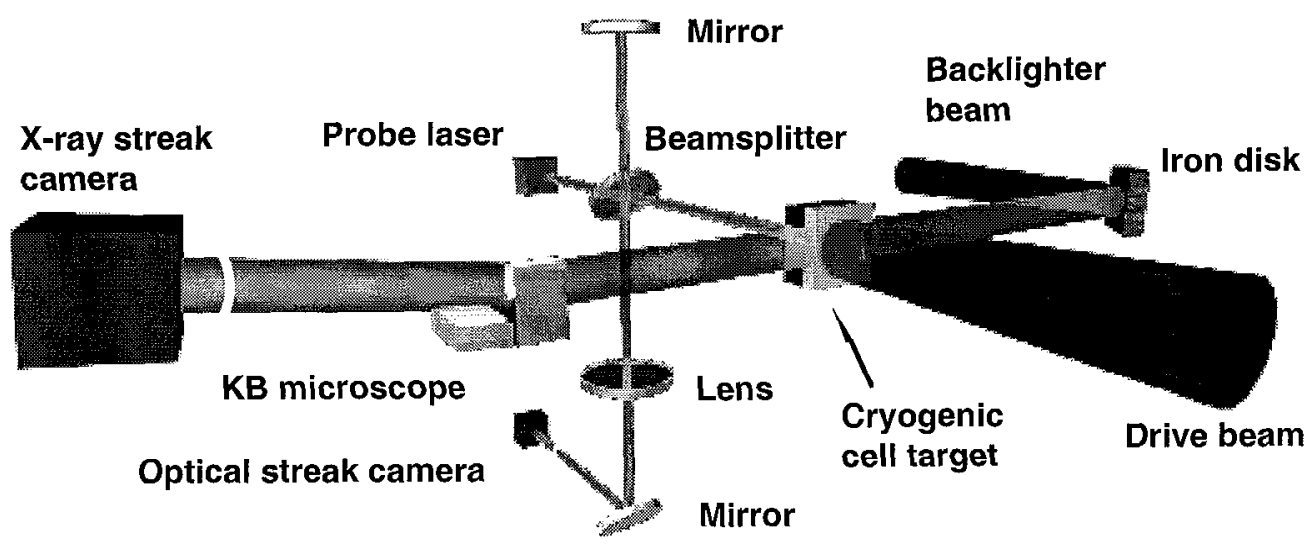

Figure 3. Schematic diagram of the experimental setup for simultaneous side-on radiography and end-on interferometry of a cryogenic tOS target. 
A schematic of the cryogenic target cell is shown in Fig. 2. Liquid $\mathrm{D}_{2}$ was contained in a 1-mmdiameter, $0.45-\mathrm{mm}$-long cylindrical cell machined into copper. One end of the cell was sealed with an $\mathrm{Al}$ or Be disk that served as the shock pusher; the opposite end of the cell was sealed with a 0.5 -mm-thick sapphire window. The pusher was $100-250 \mu \mathrm{m}$ thick depending on the experiment and had a rear-side mean surface roughness of $30 \mathrm{~nm} \mathrm{rms} \mathrm{(} 90 \mathrm{~nm}$ for the Be pushers). The pusher was coated with polystyrene to prevent direct laser ablation of the pusher, minimizing $\mathrm{x}$-ray emission and consequent preheat of the pusher from $x$ rays produced in the plasma. To accommodate transverse radiography, a $500-\mu \mathrm{m}$-diameter window was drilled into each side of the cell and sealed with a $5-\mu \mathrm{m}$-thick beryllium foil. Liquid $\mathrm{D}_{2}$ was loaded into the cell at $19 \mathrm{~K}$; temperatures were monitored so that the initial $\mathrm{D}_{2}$ density $\rho_{o}$ of about $0.170 \mathrm{~g} / \mathrm{cm}^{3}$ was known for each experiment with an uncertainty of less than $0.1 \%$.

The experimental layout used is shown in Fig. 3. One beam of the Nova laser $(\lambda=527 \mathrm{~nm})$ was focused at normal incidence onto the target, ablating the polystyrene layer and driving a shock wave through the pusher and into the $\mathrm{D}_{2}$. The beam was optically smoothed to produce a flat-top irradiance profile. A second Nova beam irradiated an iron backlighter foil, providing a source of $x$ rays for transverse radiography. Radiography was accomplished using a high resolution $x$-ray Kirkpatrick-Baez $(\mathrm{KB})$ microscope with the image recorded on a streak camera.

An example of a streaked transverse radiograph of shock-compressed $D_{2}$ is shown in Fig. 4. The drive irradiance was covered $8 \mathrm{~ns}$. The pusher is opaque and the liquid transparent so the interface is the boundary between the light and dark regions. In the figure, the interface is stationary prior to 2 ns. At 2 ns the laser-driven shock crosses the interface and the pusher surface accelerates to a steady speed, $U_{p}$. The shock front, moving ahead of the interface, appears as the dark line (visible because of refraction at the shock front). The shock speed is steady until $\sim 6 \mathrm{~ns}$ when a stronger shock enters the $\mathrm{D}_{2}$; no data after $6 \mathrm{~ns}$ were used. The shock and particle speeds can be evaluated from the slopes; $\mathrm{Eq}(1)$ then determines $P$ and $\rho$.

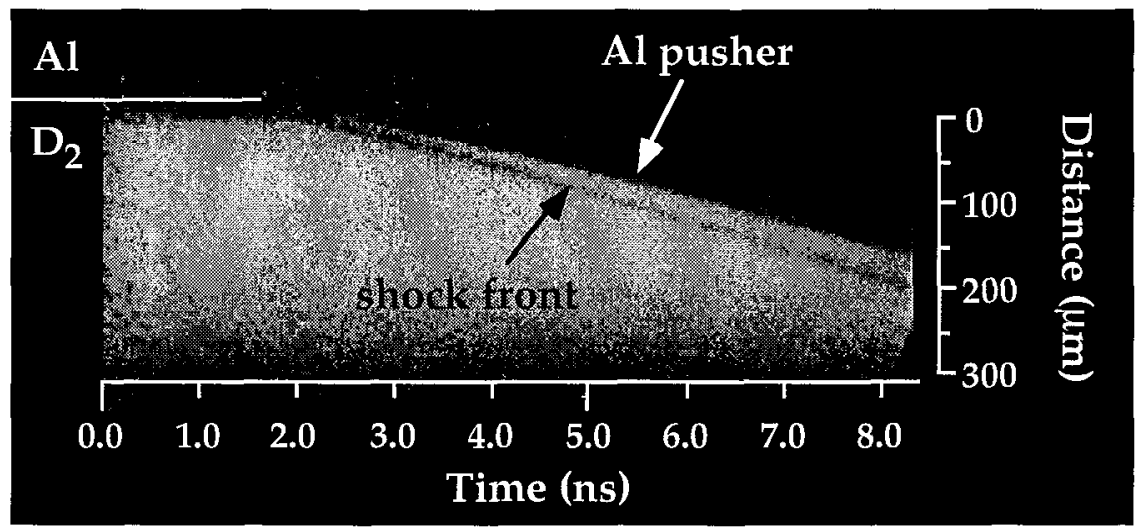

Figure 4. A side-on streak radiograph of shocked liquid $\mathrm{D}_{2}$.

Fig. 5 shows the experimental $D_{2}$ single shock Hugoniot data [23,24] along with a number of $D_{2}$ principal Hugoniot curves: the SESAME equation of state table[15]; path integral quantum Monte Carlo (QMC) simulations [19]; the Saumon-Chabrier model (SC) [17]; and a simple linear-mixing model due to Ross [22]. The data occupy a region of phase space previously experimentally unexplored. Most of the EOS model Hugoniots in Fig. 5 do not replicate the data, nor is there much agreement between models near 1 Mbar. At the lowest compression, our data are in agreement with earlier gas gun results[25]; at higher compressions, the data deviate from most predictions. The data show a significantly enhanced compressibility compared to the SESAME, but are similar to $\mathrm{SC}$ and the Ross models. 


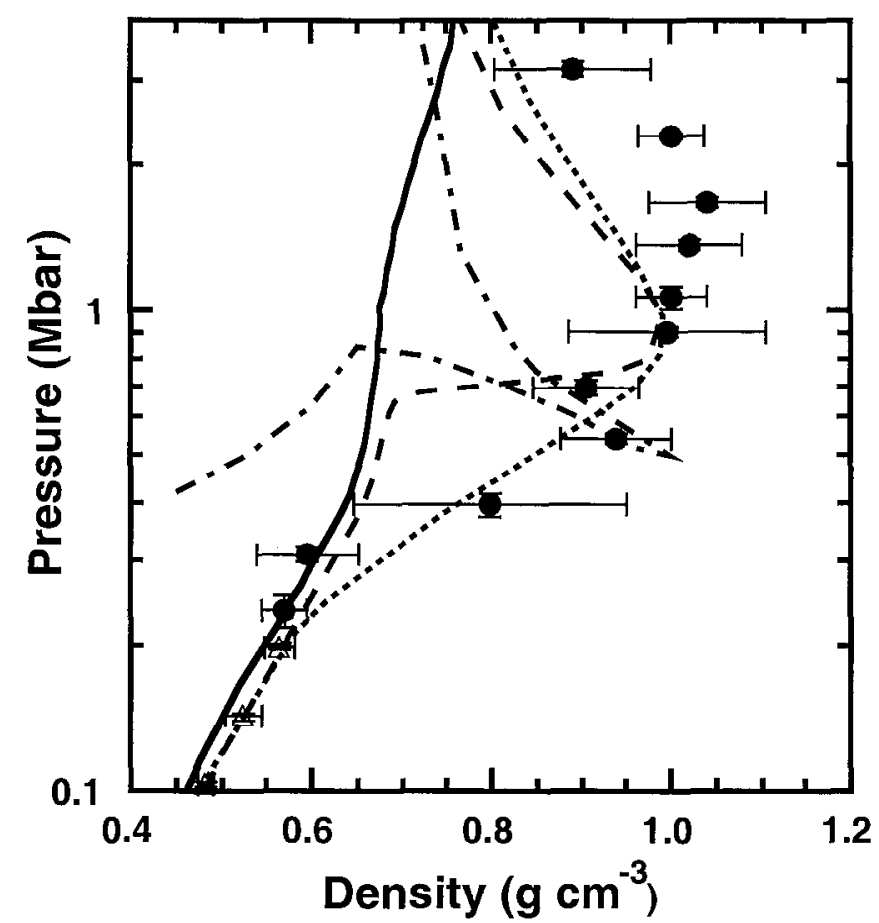

Figure 5. Nova data $[23,24]$ and gas gun data [25] (triangles) compared with predicted $\mathrm{D}_{2}$ principal Hugoniots: SESAME [15] (solid); quantum Monte Carlo simulations [19] (dot-dash); Saumon and Chabrier [17] (dash) and a linear-mixing model [22] (dots).

The SC Hugoniot differs from our data primarily between 0.4 and 0.7 Mbar, a region where they predict a first order phase transition (the so-called plasma phase transition or PPT). QMC simulations also show a PPT on the Hugoniot but at a lower pressure. A primary assumption of the Ross model is that the EOS can be fit by a simple interpolation between the insulating and conducting states and therefore precludes the existence of a PPT. That model contains one empirical parameter that was fit to gas-gun temperature data [25]. Compared to SESAME, Ross predicts more energy absorbed in the transition from a molecular insulator to an atomic conductor leading to lower temperatures and higher densities near 1 Mbar. The data show no evidence of a PPT.

The sapphire window at the rear of the cell provided a port with which to view the rear of the pusher and the shock in a face-on configuration. To assess sample preheat a Michelson interferometer was used to measure the thermal expansion of the pusher rear surface. Expansion of the rear pusher surface prior shock arrival - preheal - produced fringe motion al the delector. Measurements showed that the temperature of the pusher/ $\mathrm{D}_{2}$ interface was maintained below our detection limit.

For some experiments the Michelson interferometer was replaced with a velocity interferometer [26]. This is a technique that measures the Doppler shift of light reflected from a moving surface; the recorded fringe shift is directly proportional to the Doppler shift and, therefore, to the velocity of the reflecting surface. The temporal resolution is less than 0.1 fringe providing $\sim 1 \%$ accuracy in $U_{s}$ if the shock front is sufficiently reflecting. In addition to $U_{s}$, the velocity interferometer also supplies instantaneous measurements of the (single-probe-laser-wavelength) reflectivity of the shock front. At low shock pressures ( $0.2 \mathrm{Mbar})$, the reflectivity is a few percent. However, above $0.55 \mathrm{Mbar}$ the measured reflectivities are around $60 \%$, characteristic of a metal. [27] 
The temperature of the shock front was also determined by employing an oplical pyrometer in place of the interferometers. The pyrometer measured emission in the six bands. Fitting the emission curves to a grey-body formula determined the temperature [28]. The temperature of the shocked $\mathrm{D}_{2}$ is $\sim 1.5 \mathrm{eV}$ at $1 \mathrm{Mbar}$. This is much less than the ionization potential so that the metallike shocked- $\mathrm{D}_{2}$ reflectivity is due to free electrons produced by a combination of density and thermal effects, or pressure ionization. The temperature is also much less than the $15 \mathrm{eV}$ Fermi temperature so the term metal is appropriate. Fig. 6 displays temperature measurements.

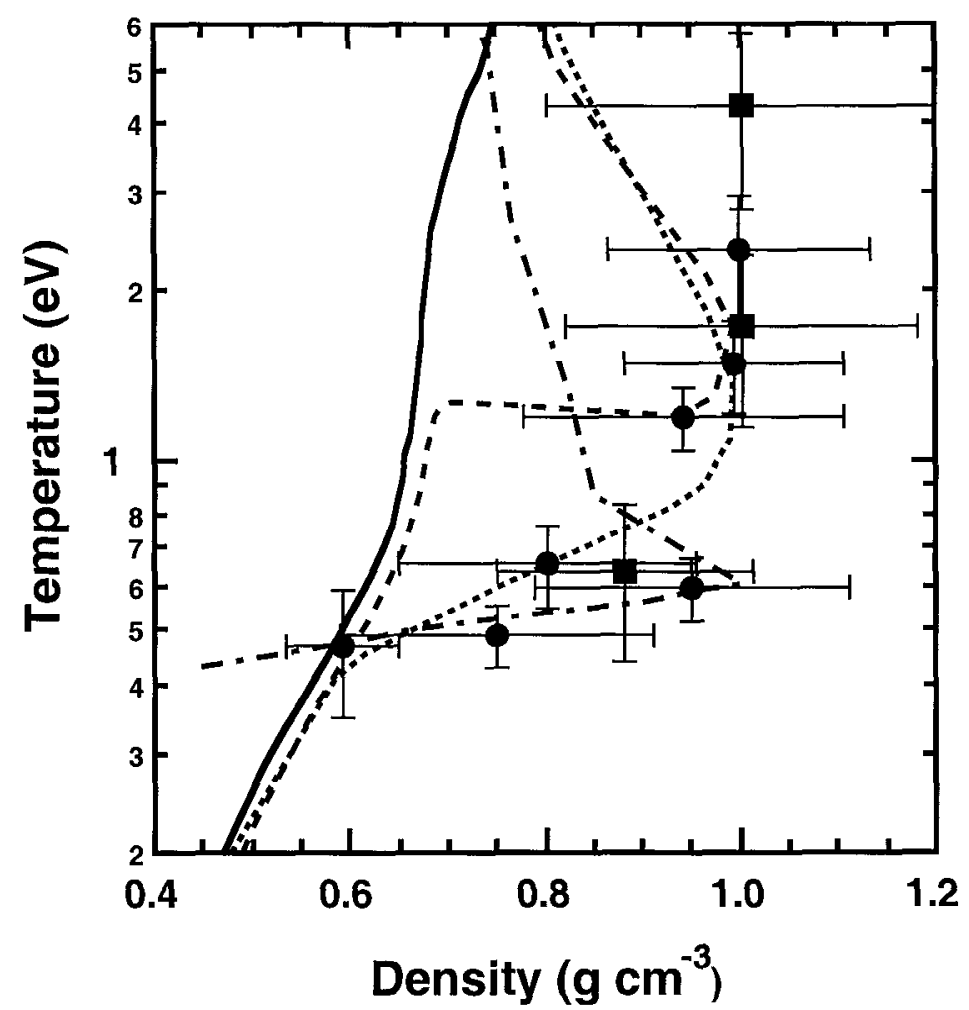

Figure 6. Shock temperature along the Hugoniot. Labels are same as Fig. 5.

\section{CONCLUSIONS}

There are two important consequences of the hydrogen experiments and the predicted enhanced compressibility of hydrogen isotopes. First, higher compressibility suggests that the mass distribution in the Jovian plancts (i.e. ncar $\sim 0.8$ of the radius of Jupiter) is different than current models predict. Second, the more compressible EOS of hydrogen leads directly to higher fuel densities in ICF targets; this offers the possibility of higher performance and improved margin for ICF ignition capsules. These are practical, but very important consequences. A fundamental outcome of this and further experimental work on the EOS of hydrogen will be a far more refined theoretical knowledge of the high pressure equation of state of the most abundant element in the universe. Mbar data, were obtained in the laboratory in a regime where the theory is extremely difficult, are a necessary component of that reformulation.

High energy lasers can be used to explore regimes that have heretofore been inaccessible in the laboratory. Hydrogen isotope EOS at high energy density is one example. We are continuing to 
experiment with other materials, including high pressure water and carbon, that are important constituents in the solar system and beryllium and plastics that are crucial to the success of inertial confinement fusion.

\section{REFERENCES}

1. C. G. M. van Kessel and R. Sigel, Phys. Rev. Lett. 33, 1020 (1974); L. R. Veeser and S. C. Solem, Phys. Rev. Lett. 40, 1391 (1978); R. J. Trainor, J. W. Shaner, J. M. Auerbach, and N. C. Holmes, Phys. Rev. Lett. 42, 1154 (1979).

2. E. M. Campbell, Laser Part. Beams 9, 209 (1991).

3. S. Fu, Y. Gu, J. Wu, and S. Wang, Phys. Plasmas 2, 3461 (1995).

4. M. Koenig et al., Phys. Rev. Lett., 74, 2260 (1995).

5. A. M. Evans et al., Lasers Part. Beams 14, 113 (1996).

6. A. Benuzzi et al., Phys. Rev. E 54, 2162 (1996).

7. R. Cauble et al., Phys. Rev. Lett. 70, 2102 (1993)

8. R. Cauble et al., Phys. Rev. Lett. 74, 3816 (1995)

9. Y. B. Zel'dovich and Y. P. Raizer, Physics of Shock Waves and High-Temperature Hydrodynamic Phenomena (Academic Press, New York, 1966).

10. C. E. Ragan III, Phys. Rev. A 25, 3360 (1982); W. J. Nellis et al., Phys. Rev. Lett. 60, 1414 (1988).

11. R. Cauble et al.,"Phys. Rev. Lett. 80, 1248 (1998).

12. R. Smoluchowski, Nature 215, 691 (1967); W. B. Hubbard, Science 214, 145 (1981); G. Chabrier, D. Saumon, W. B. Hubbard, J. I. Lunine, Astrophys. J. 391, 817 (1992); W. J. Nellis, M. Ross, N. C. Holmes, Science 269, 1249 (1995).

13. G. Chabrier and I. Baraffe, Astron. Astrophys. 327, 1039 (1997).

14. S. W. Haan et al., Phys. Plasmas 2, 2480 (1995); J. D. Lindl, Phys. Plasmas 2, 3933 (1995); S. Nakai and H. Takabe, Rep. Prog. Phys. 59, 1071 (1996).

15. G. I. Kerley, "A Theoretical Equation of State for Deuterium," Los Alamos Laboratory Report LA-4776 (1972); G. Kerley, J. Chem. Phys. 73, 460 (1980).

16. M. Ross, F. H. Rcc, D. A. Young, J. Chem. Phys. 79, 1487 (1983).

17. D. Saumon and G. Chabrier, Phys. Rev A 44, 5122 (1991); 46, 2084 (1992); Phys. Rev. Lett. 62, 2397 (1989).

18. H. Reinholz, R. Redmer, S. Nagel, Phys. Rev. E 52, 5368 (1995).

19. W. R. Magro, D. M. Ceperley, C. Pierleoni, B. Bernu, Phys. Rev. Lett. 76, 1240 (1996).

20. F. J. Rogers, Astrophys. J. 310, 723 (1986); F. J. Rogers, F. J. Swenson, C. A. Iglesias, Astrophys. J. 456, 902 (1996).

21. T. J. Lenosky, J. D. Kress, L. A. Collins, Phys. Rev. B 56, 5164 (1997).

22. M Ross, 58, 669 Phys. Rev. B (1998).

23. L. B. Da Silva et al. Phys. Rev. Lett. 78, 483 (1997); R. Cauble et al. Phys. Plasmas 4, 1857 (1997); G. W. Collins et al., Phys. Plasmas 5, 1864 (1998).

24. G. W. Collins et al., Science $\mathbf{2 8 1}$ (1998) 1178

25. W. J. Nellis, A. C. Mitchell, M. van Thiel, G. J. Devine, R. J. Trainor, and N. Brown, J. Chem. Phys. 79, 1480 (1983); N. C. Holmes, M. Ross, and W. J. Nellis, Phys. Rev. B 52, 15835 (1995).

26. L. M. Barker and R. E. Hollenbach, J. Appl. Phys. 43, 4669 (1972); P. M. Celliers et al. Appl. Phys. Lett. 73, 1320 (1998).

27. P. M. Celliers et al, submitted to Phys. Rev. Lett. (1998).

28. G. W. Collins et al., submitted to Phys. Rev. Lett. (1998).

29. D. Saumon, G. Chabrier, and H. M. Van Horn, Astrophys. J. Supp. 99, 713 (1995).

30. W. B. Hubbard et al, Phys. Plasmas 4, 2011 (1997).

31. This work was performed under the auspices of the U.S. Department of Energy by Lawrence Livermore National Laboratory under contract no. W-7405-Eng-48. 